\title{
26 Research Soure \\ Mental Health In Myasthenia Gravis Patients And Its Impact On Caregiver Burden
}

Derin Marbin ( $\nabla$ derin.marbin@charite.de)

Charité - University Medicine Berlin

\section{Sophie K. Piper}

Charité - University Medicine Berlin

\section{Sophie Lehnerer}

Charité - University Medicine Berlin

\section{Ulrike Harms}

Charité - University Medicine Berlin

\section{Andreas Meisel}

Charité - University Medicine Berlin

\section{Research Article}

Keywords: myasthenia, caregiver burden, quality of life, depression, anxiety, mental health

Posted Date: February 21 st, 2022

DOI: https://doi.org/10.21203/rs.3.rs-1320921/v1

License: (c) (1) This work is licensed under a Creative Commons Attribution 4.0 International License. Read Full License 


\section{Abstract}

Background: Psychiatric comorbidities are relevant in patients with Myasthenia gravis (MG). Also, MG patients experience a reduced health related quality of life (HRQoL). We aimed to quantify the impact of depression as well as self-perceived MG severity on HRQoL and caregivers' burden.

Methods: In this cross-sectional study, we used a survey encompassing demographic, disease-related information and standardized questionnaires to assess depression, anxiety, HRQoL (MG Quality of Life scale; MG-QoL15) and caregiver burden (Burden Scale for Family Caregivers; BSFC).

Results: Data from 1399 participating patients (96\%) and 1042 caregivers (65\%) were eligible for further analysis. Depression and anxiety disorder were indicated by $31 \%$ and $36 \%$ of patients. Self-reported MG severity and MG-QoL15 scores were strongly associated (estimated marginal means for severe vs. mild MG severity= $1895 \% \mathrm{Cl}[16 ; 21] ; \mathrm{p} \leq 0.001)$. Adjusting for depression decreased the estimated strength of this association (estimated marginal means for severe vs. mild MG severity= 15 [13; 17]; $p \leq 0.001$ ). Caregiver burden is associated to MG disease severity (estimated marginal means for severe vs. mild MG severity $=0.16[0.13 ; 0,19) ; p \leq 0.001)$ and also negatively influenced by depression (estimated marginal means $=0.12[0.09 ; 0.15])$.

Conclusion: Depression and anxiety disorder in MG are frequent. Beyond MG severity, depression has negative effects on HRQoL as well as on caregivers' burden. Diagnosis and treatment of psychiatric comorbidities should be considered as an important element in MG care. Screening tools for mental health conditions should be implemented at least in specialized MG centers.

\section{Introduction}

Myasthenia gravis (MG) is a chronic, autoimmune-mediated disease of the neuromuscular junction with fluctuating weakness in skeletal muscles (1). The median prevalence is 15 per 100,000 (2). Symptoms can encompass ocular symptoms such as diplopia and ptosis, faciopharyngeal weakness like dysphagia and dysarthrophonia as well as generalized weakness affecting limb, neck, and torso musculature. Almost $20 \%$ of patients suffer from a life-threatening myasthenic crisis at least once during the course of disease. It is characterized by a respiratory insufficiency, requiring intensive care treatment with mechanical ventilation $(3,4)$. Although significant advances in therapy prolonged overall life expectancy, a reduced Health-Related Quality of Life (HRQOL) is reported, especially in MG patients with bulbar and generalized symptoms (5). There is inconsistent data about the reduction of psychological domains in the HRQoL $(5,6)$. Activities of daily life (AdL) are also reduced in MG patients (7). The impact of MG itself as well as its comorbidities including those affecting mental health have become an important focus in research.

Psychiatric comorbidities like depression, anxiety disorder, or posttraumatic stress disorder (PTSD) are highly prevalent in smaller cohorts of MG patients, with prevalence of depression ranging from $14-58 \%$ $(8,9)$. Depression and other factors like illness stability and impairments have been identified as 
important factors affecting the HRQoL (10), which needs to be considered in the treatment of MG patients (11). So far, the detection and adequate treatment of mood disorders in MG patients seems insufficient $(12,13)$. However, the impact of MG severity and related mental disorders on the burden of disease is limited. Importantly, their effects on caregivers' burden are unknown. We hypothesize that the prevalence of depression, anxiety and PTSD is not only higher amongst MG patients, but also underdiagnosed. We further hypothesize that the self-perceived MG severity as well as comorbid depression have a significant impact on patients' Health-Related Quality of Life and caregivers' burden.

Thus, we investigated the prevalence and underdiagnosis of psychiatric comorbidities (depression, anxiety disorder, and PTSD) in MG patients. We aimed to quantify the effect of self-reported disease severity and depression on patients HRQoL and caregivers' burden.

\section{Methods}

\section{Patients}

Study participants were recruited between April and December 2017. In cooperation with the German Myasthenia Society (self-help group for MG in Germany; DMG) we asked all 3280 members via postal mail to fill out a questionnaire. The study participants were instructed to return their completed questionnaire in a prepaid envelope with no identity information to ensure the anonymity of the survey. Further subjects were recruited in our MG outpatient clinic ("Integrated MG Center") at the Charité University Hospital Berlin by our physicians. Before enrollment, all patients had to confirm that they did not already participate in this study via the DMG and gave written informed consent. No refund was given.

All participants were asked to encourage their caregivers to provide information by an additional questionnaire assessing the caregiver burden. Exclusion criteria were age under 18, missing diagnosis of MG, and insufficiently completed questionnaires, defined a priori as less than fifty percent of all items answered. All data were de-identified for analysis. The study has been approved by the ethics committee of Charité University Medicine Berlin (reference number EA1/028/17), and registered at clinicaltrials.gov (NCT03205306). All research was performed in accordance to the Helsinki Declaration.

\section{Parameters and Scores}

The questionnaires contained demographic data like gender, age, marital status, and educational status. Relevant disease-related information such as current disease severity (low, medium, high), antibody status, age at onset of myasthenia-related symptoms and time of diagnosis, comorbidities (autoimmune, cardiac or psychiatric (depression, anxiety, PTSD, other)), medication, history of myasthenic crisis and exacerbation.

Psychiatric comorbidities and burden of disease were assessed by using following validated German versions of standardized and self-administrated questionnaires: Hospital Anxiety and Depression Scale 
(HADS), MG Quality of Life Scale (MG-QoL15), short DSM-IV screening scale for PTSD (PTSD-7), Freiburger Fragebogen zur Krankheitsverarbeitung (FKV; not available in English) and Burden Scale for Family Caregivers (BSFC).

The HADS is a screening instrument with a high validity and reliability (sensitivity and specificity 0.8 ; Cronbach's alpha for Anxiety: 0.83 and for Depression: 0.82 ) to detect depression and anxiety amongst patients with somatic diseases (14). It contains seven items for each anxiety and depression. The cut-off score is seven, indicating cases above cut-off in gradation mild (8-10), moderate (11-14), and severe (>14). The MGQOL15 is a short version of the sixty items MGQOL.(15) It is a disease-specific healthrelated QoL scale, with lower scores indicating a higher HRQoL.

We asked each patient with a myasthenic crisis (with ICU submission) or exacerbation throughout the disease to fill out the PTSD-7 $(16,17)$. A sum score of $\geq 4$ implies a PTSD with a sensitivity of $80 \%$ and specificity of $97 \%$, reliability is also high (Cronbach's alpha 0.90$)(16)$. To investigate coping strategies, we used the FKV questionnaire, assessing different dimensions of coping mechanisms, e.g. cognition, emotion and behavior (18).

To examine perceived caregivers' burden, we used the short version of the BSFC including 10 domains such as reduced life satisfaction, physical exhaustion, conflicting demands, or affection of their health by caregiving. Cronbach's alpha for the complete scale was 0.92 (19).

\section{Statistical analysis}

For data transfer and analysis, we used IBM SPSS Statistics 25 and STATA IC 14. We compared sociodemographic characteristics, questionnaire scores, and clinical characteristics between the myasthenia subgroups (disease severity) using chi-square test, or Kruskal-Wallis-test, where indicated.

General linear models were used to estimate the association between self-reported disease severity as independent variable and MG-QoL15 and caregiver burden as dependent variables, respectively. Models were stepwise adjusted for potential effect mediation by depression according to HADS scores $(1:<8 ; 2$ : $8-10 ; 3: 11-14 ; 4:>14)$, as well as the potential confounding factors gender, age, education, marital status, myasthenic crisis/exacerbation, duration of disease, disease latency and treatment escalation (IVIG/Rituximab) as well as a history auf other autoimmune disease or cardiovascular disease as binary variables.

Continuous variables with a right-skewed distribution, namely the duration of disease and the disease latency were log transformed before entered into the model. Adjusted $\mathrm{R}^{2}$ values are given for goodness of fit. A two-sided significance level of $a=0.05$ was considered. No adjustment for multiple testing was applied and all p-values constitute exploratory data analysis.

\section{Results}


The questionnaires were filled out by 1451 patients yielding an overall response rate of $44.2 \%$ (Table 1 ). According to the exclusion criteria 52 questionnaires were excluded. Caregivers' response rate was $47 \%$ (1596). Incompletely filled out questionnaires were excluded, $65 \%$ of the questionnaires were eligible for further analyses (1042). The median duration of MG was 9 years (IQR 4-18). Median age of MG onset was 39 years (IQR 24-57) in woman and 62 years (IQR 51-70) in men. Correspondingly, the median age of diagnosis was 44 years (IQR 28-60) in woman and 62 years (IQR 51-70) in men. Less than half of the patients had undergone thymectomy $(42.9 \%)$, with $67.7 \%$ of them being female patients. Intake of regular and delayed-release pyridostigmine was reported by and $63.5 \%$ and $39.0 \%$. The most commonly used immunosuppressant was azathioprine (43.8\%), steroids were taken by $23.4 \%$ of the participants.

Escalation therapy with Rituximab and IVIG were reported by $3.4 \%$ and $8.2 \%$, respectively. Experience of at least one myasthenic crisis was reported by $17.2 \%$ of patients, whereas $22.8 \%$ reported exacerbations of MG. The lead institution in MG care was a certified center for MG care ("integrated myasthenia center") for $20.7 \%$ of patients and a neurological outpatient clinic of a university hospital for $30.9 \%$. The majority of patients $(60.8 \%)$ also visited a registered neurologist or general practitioner. The most abundant somatic comorbidities, cardiovascular and autoimmune disorders, were reported by $18.1 \%$ and $18.9 \%$, respectively (Table 1). Among autoimmune disorders, rheumatoid arthritis, hypothyroidism, Hashimoto's thyroiditis, psoriasis, and systemic lupus erythematosus were most often reported (data not shown). 
Table 1

Characteristics of MG patients. $\mathrm{N}=1399$

\begin{tabular}{|c|c|c|c|c|c|c|}
\hline \multirow{2}{*}{\multicolumn{2}{|c|}{$\begin{array}{l}\text { self-reported MG } \\
\text { severity, } n(\%) n=13\end{array}$}} & Total & Low & Moderate & High & $p$-value \\
\hline & & 1399 & $\begin{array}{l}469 \\
(35.3)\end{array}$ & $\begin{array}{l}690 \\
(51.9)\end{array}$ & $\begin{array}{l}170 \\
(12.8)\end{array}$ & \\
\hline \multicolumn{2}{|l|}{$\begin{array}{l}\text { age, median (IQR). } \\
n=1329\end{array}$} & $\begin{array}{l}67 \\
(54- \\
76)\end{array}$ & $\begin{array}{l}66 \\
(54- \\
75)\end{array}$ & $\begin{array}{l}66(54- \\
76)\end{array}$ & $\begin{array}{l}68(55- \\
77)\end{array}$ & $0.376^{\mathrm{a}}$ \\
\hline $\begin{array}{l}\text { age at onset of } \\
\text { myasthenic }\end{array}$ & \multirow{3}{*}{$\begin{array}{l}\text { male } \\
\text { female }\end{array}$} & $\begin{array}{l}62 \\
(51-\end{array}$ & $\begin{array}{l}61 \\
(51-\end{array}$ & $\begin{array}{l}63(52- \\
70)\end{array}$ & $\begin{array}{l}60,5(49- \\
70)\end{array}$ & $0.498^{a}$ \\
\hline \multirow{2}{*}{$\begin{array}{l}\text { symptoms, median } \\
\text { (IQR). } n=1257\end{array}$} & & 70) & 69) & 41 (25- & $34(20-$ & $0.148^{a}$ \\
\hline & & $\begin{array}{l}39 \\
(24- \\
57)\end{array}$ & $\begin{array}{l}37 \\
(24- \\
55)\end{array}$ & 58) & $56)$ & \\
\hline \multirow{2}{*}{$\begin{array}{l}\text { Gender } \\
n=1327\end{array}$} & male & $\begin{array}{l}605 \\
(45.6)\end{array}$ & $\begin{array}{l}248 \\
(53.1)\end{array}$ & $\begin{array}{l}280 \\
(40.6)\end{array}$ & $77(45.3)$ & $<0.001^{b}$ \\
\hline & female & $\begin{array}{l}722 \\
(54.4)\end{array}$ & $\begin{array}{l}219 \\
(46.9)\end{array}$ & $\begin{array}{l}410 \\
(59.4)\end{array}$ & $93(54.7)$ & \\
\hline \multirow{3}{*}{$\begin{array}{l}\text { education degree } \\
n=1245\end{array}$} & basic & $\begin{array}{l}62 \\
(5.0)\end{array}$ & $\begin{array}{l}19 \\
(4.3)\end{array}$ & $33(5.2)$ & $10(6.2)$ & $0.014^{a}$ \\
\hline & secondary & $\begin{array}{l}778 \\
(62.5)\end{array}$ & $\begin{array}{l}260 \\
(58.4)\end{array}$ & $\begin{array}{l}422 \\
(66.1)\end{array}$ & $96(59.3)$ & \\
\hline & higher & $\begin{array}{l}405 \\
(32.5)\end{array}$ & $\begin{array}{l}166 \\
(37.3)\end{array}$ & $\begin{array}{l}183 \\
(28.7)\end{array}$ & $56(34.6)$ & \\
\hline \multirow{3}{*}{$\begin{array}{l}\text { marital status } \\
n=1325\end{array}$} & married/partnership & $\begin{array}{l}986 \\
(74.4)\end{array}$ & $\begin{array}{l}358 \\
(76.3)\end{array}$ & $\begin{array}{l}497 \\
(72.3)\end{array}$ & $131(77.5)$ & $0.225^{\mathrm{a}}$ \\
\hline & single & $\begin{array}{l}106 \\
(8.0)\end{array}$ & $\begin{array}{l}38 \\
(8.1)\end{array}$ & $61(8.9)$ & $7(4.1)$ & \\
\hline & divorced/widowed & $\begin{array}{l}233 \\
(17.6)\end{array}$ & $\begin{array}{l}73 \\
(15.6)\end{array}$ & $\begin{array}{l}129 \\
(18.8)\end{array}$ & 31 (18.3) & \\
\hline \multicolumn{2}{|c|}{$\begin{array}{l}\text { duration of MG disease in years, median } \\
(\text { IQR). } n=1329\end{array}$} & $\begin{array}{l}9(4- \\
18)\end{array}$ & $\begin{array}{l}10(4- \\
18)\end{array}$ & $9(4-18)$ & $11(5-22)$ & $0.041^{a}$ \\
\hline \multicolumn{2}{|c|}{$\begin{array}{l}\text { latency of diagnosis in years, median (IQR). } \\
n=1250\end{array}$} & $\begin{array}{l}0(0- \\
1)\end{array}$ & $\begin{array}{l}0(0- \\
1)\end{array}$ & $0(0-2)$ & $0(0-2)$ & $<0.001^{a}$ \\
\hline \multicolumn{2}{|l|}{$\begin{array}{l}\text { myasthenic crisis. } \\
n=1384\end{array}$} & $\begin{array}{l}240 \\
(17.2)\end{array}$ & $\begin{array}{l}44 \\
(9.4)\end{array}$ & $\begin{array}{l}116 \\
(16.8)\end{array}$ & $67(39.4)$ & $<0.001^{b}$ \\
\hline
\end{tabular}

\footnotetext{
${ }^{a}$ Kruskal-Wallis-Test, ${ }^{b}$ Chi-square test. $P$-values refer to overall group comparisons of patients with Iow, moderate and high MG severity. ICU=Intensive care unit. P-values $<0.05$ are marked in bold numbers.
} 


\begin{tabular}{|c|c|c|c|c|c|}
\hline \multirow{2}{*}{$\begin{array}{l}\text { self-reported MG } \\
\text { severity, } n(\%) n=1329\end{array}$} & Total & Low & Moderate & High & \multirow[t]{2}{*}{$p$-value } \\
\hline & 1399 & $\begin{array}{l}469 \\
(35.3)\end{array}$ & $\begin{array}{l}690 \\
(51.9)\end{array}$ & $\begin{array}{l}170 \\
(12.8)\end{array}$ & \\
\hline $\begin{array}{l}\text { myasthenic } \\
\text { exacerbation. } n=1384\end{array}$ & $\begin{array}{l}319 \\
(22.8)\end{array}$ & $\begin{array}{l}79 \\
(16.8)\end{array}$ & $\begin{array}{l}194 \\
(28.1)\end{array}$ & $46(27.1)$ & $<0.001^{b}$ \\
\hline $\begin{array}{l}\text { other autoimmune } \\
\text { diseases. }\end{array}$ & $\begin{array}{l}264 \\
(18.9)\end{array}$ & $\begin{array}{l}81 \\
(17.3)\end{array}$ & $\begin{array}{l}155 \\
(22.5)\end{array}$ & $28(16.5)$ & $0.047^{b}$ \\
\hline \multicolumn{6}{|l|}{$n=1280$} \\
\hline $\begin{array}{l}\text { cardiovascular } \\
\text { diseases. } n=1289\end{array}$ & $\begin{array}{l}253 \\
(18.1)\end{array}$ & $\begin{array}{l}72 \\
(15.4)\end{array}$ & $\begin{array}{l}139 \\
(20.1)\end{array}$ & $42(24.7)$ & $0.010^{b}$ \\
\hline Thymectomy. n=1302 & $\begin{array}{l}600 \\
(42.9)\end{array}$ & $\begin{array}{l}212 \\
(45.2)\end{array}$ & $\begin{array}{l}303 \\
(44.0)\end{array}$ & $85(50.0)$ & $0.295^{b}$ \\
\hline $\begin{array}{l}\text { Pyridostigmine. } \\
n=1328\end{array}$ & $\begin{array}{l}888 \\
(63.5)\end{array}$ & $\begin{array}{l}253 \\
(54.0)\end{array}$ & $\begin{array}{l}505 \\
(73.2)\end{array}$ & $\begin{array}{l}130 \\
(76.5)\end{array}$ & $<0.001^{b}$ \\
\hline $\begin{array}{l}\text { Delayed-release } \\
\text { Pyridostigmine retard. } \\
n=1329\end{array}$ & $\begin{array}{l}545 \\
(39.0)\end{array}$ & $\begin{array}{l}125 \\
(26.7)\end{array}$ & $\begin{array}{l}341 \\
(49.4)\end{array}$ & $79(46.5)$ & $<0.001^{b}$ \\
\hline Azathioprine. $n=1329$ & $\begin{array}{l}613 \\
(43.8)\end{array}$ & $\begin{array}{l}194 \\
(41.4)\end{array}$ & $\begin{array}{l}360 \\
(52.2)\end{array}$ & $59(34.7)$ & $<0.001^{b}$ \\
\hline Steroids. $n=1251$ & $\begin{array}{l}328 \\
(23.4)\end{array}$ & $\begin{array}{l}71 \\
(15.2)\end{array}$ & $\begin{array}{l}193 \\
(28.0)\end{array}$ & $64(37.6)$ & $<0.001^{b}$ \\
\hline $\begin{array}{l}\text { Mycophenolate } \\
\text { Mofetil. } n=1329\end{array}$ & $\begin{array}{l}126 \\
(9.0)\end{array}$ & $\begin{array}{l}27 \\
(5.8)\end{array}$ & $68(9.9)$ & $31(18.2)$ & $<0.001^{b}$ \\
\hline Methotrexate. $n=1329$ & $\begin{array}{l}53 \\
(3.8)\end{array}$ & $\begin{array}{l}21 \\
(4.5)\end{array}$ & $20(2.9)$ & $12(7.1)$ & $0.037^{b}$ \\
\hline $\begin{array}{l}\text { Ciclosporine A. } \\
n=1329\end{array}$ & $\begin{array}{l}15 \\
(1.1)\end{array}$ & $\begin{array}{l}0 \\
(0.0)\end{array}$ & $12(1.7)$ & $3(1.8)$ & $0.016^{b}$ \\
\hline Rituximab. n=1329 & $\begin{array}{l}47 \\
(3.4)\end{array}$ & $\begin{array}{l}5 \\
(1.1)\end{array}$ & $21(3.0)$ & $21(12.4)$ & $<0.001^{b}$ \\
\hline $\begin{array}{l}\text { Intravenous } \\
\text { immunoglobulin. } \\
n=1329\end{array}$ & $\begin{array}{l}115 \\
(8.2)\end{array}$ & $\begin{array}{l}11 \\
(2.3)\end{array}$ & $57(8.3)$ & $47(27.6)$ & $<0.001^{b}$ \\
\hline $\begin{array}{l}\text { MGQOL15 score, } \\
\text { median (IQR). } n=1037\end{array}$ & $\begin{array}{l}12(4- \\
26)\end{array}$ & $\begin{array}{l}4(1- \\
10)\end{array}$ & $18(9-29)$ & $\begin{array}{l}25(9- \\
385)\end{array}$ & $<0.001^{a}$ \\
\hline $\begin{array}{l}\text { Sleep difficulties. } \\
n=1183\end{array}$ & $\begin{array}{l}881 \\
(63.0)\end{array}$ & $\begin{array}{l}247 \\
(52.7)\end{array}$ & $\begin{array}{l}512 \\
(75.2)\end{array}$ & $\begin{array}{l}122 \\
(71.8)\end{array}$ & $<0.001^{b}$ \\
\hline
\end{tabular}

\footnotetext{
${ }^{a}$ Kruskal-Wallis-Test, ${ }^{b}$ Chi-square test. $P$-values refer to overall group comparisons of patients with Iow, moderate and high MG severity. ICU=Intensive care unit. P-values $<0.05$ are marked in bold numbers.
} 


\begin{tabular}{|c|c|c|c|c|c|}
\hline \multirow{2}{*}{$\begin{array}{l}\text { self-reported MG } \\
\text { severity, } n(\%) n=1329\end{array}$} & Total & Low & Moderate & High & \multirow[t]{2}{*}{$p$-value } \\
\hline & 1399 & $\begin{array}{l}469 \\
(35.3)\end{array}$ & $\begin{array}{l}690 \\
(51.9)\end{array}$ & $\begin{array}{l}170 \\
(12.8)\end{array}$ & \\
\hline $\begin{array}{l}\text { Sexual dysfunction. } \\
n=1209\end{array}$ & $\begin{array}{l}755 \\
(54.0)\end{array}$ & $\begin{array}{l}201 \\
(42.9)\end{array}$ & $\begin{array}{l}442 \\
(64.1)\end{array}$ & $\begin{array}{l}112 \\
(65.9)\end{array}$ & $<0.001^{b}$ \\
\hline $\begin{array}{l}\text { BSFC score, median } \\
\text { (IQR). } n=977\end{array}$ & $\begin{array}{l}3(0- \\
10)\end{array}$ & $\begin{array}{l}1(0- \\
4)\end{array}$ & $4(0-12)$ & $9(2-15)$ & $<0.001^{a}$ \\
\hline
\end{tabular}

Of all, $81.4 \%$ negated a diagnosed mental health condition, a prediagnosed depression has been reported by $11.6 \%$, and $5.7 \%$ reported an anxiety disorder (Table 2 ). In contrast, when assessing the prevalences of psychiatric comorbidities by using standardized questionnaires (HADS), we observed significant higher rates of depression (30.8\%) and anxiety disorder (35.5\%). Signs of PTSD could be identified in $11.2 \%$ of patients with a self-reported myasthenic crisis (with ICU submission) or exacerbation, which equals $4.9 \%$ of the total study population. There were no differences in the frequencies of PTSD between patient groups reporting myasthenic crisis and exacerbation. The severity of depression as well as anxiety disorder was associated significantly with higher self-reported MG severity. The prevalence of depression and PTSD was almost three times higher in patients with high compared to low MG severity $39.0 \%$ vs. $14.4 \%$ in depression; $11.0 \%$ vs. $3.8 \%$ in PTSD). Amongst MG patients with high MG severity, anxiety disorder was twice as common as in patients with a low disease severity (38.4\% vs. $19.4 \%$ ). 
Table 2

Psychiatric comorbidities in MG patients

\begin{tabular}{|c|c|c|c|c|c|c|c|}
\hline \multirow{2}{*}{$\begin{array}{l}\text { self-reported MG } \\
\text { severity, n (\%) }\end{array}$} & & \multirow[t]{2}{*}{ missing } & \multirow{2}{*}{$\begin{array}{l}\text { Total } \\
1399\end{array}$} & \multirow{2}{*}{$\begin{array}{l}\text { Low } \\
469 \\
(33.5)\end{array}$} & \multirow{2}{*}{$\begin{array}{l}\text { Moderate } \\
690 \\
(51.9)\end{array}$} & \multirow{2}{*}{$\begin{array}{l}\text { High } \\
170 \\
(12.8)\end{array}$} & \multirow[t]{2}{*}{$p$-value } \\
\hline & & & & & & & \\
\hline \multirow{5}{*}{$\begin{array}{l}\text { Depression } \\
\text { (according to } \\
\text { HADS) }\end{array}$} & $\begin{array}{l}\text { no } \\
\text { depression }\end{array}$ & 21 & $\begin{array}{l}957 \\
(69.2)\end{array}$ & $\begin{array}{l}392 \\
(85.6)\end{array}$ & $\begin{array}{l}407 \\
(60.1)\end{array}$ & $\begin{array}{l}100 \\
(61.0)\end{array}$ & $<0.001^{a}$ \\
\hline & depression & & $\begin{array}{l}421 \\
(30.8)\end{array}$ & $\begin{array}{l}66 \\
(14.4)\end{array}$ & $\begin{array}{l}270 \\
(39.9)\end{array}$ & $\begin{array}{l}64 \\
(39.0)\end{array}$ & \\
\hline & mild & & $\begin{array}{l}217 \\
(15.7)\end{array}$ & $\begin{array}{l}38 \\
(8.3)\end{array}$ & $\begin{array}{l}142 \\
(21.0)\end{array}$ & $\begin{array}{l}27 \\
(16.5)\end{array}$ & \\
\hline & moderate & & $\begin{array}{l}160 \\
(11.6)\end{array}$ & $\begin{array}{l}23 \\
(5.0)\end{array}$ & $\begin{array}{l}113 \\
(16.7)\end{array}$ & $\begin{array}{l}34 \\
(20.7)\end{array}$ & \\
\hline & severe & & $\begin{array}{l}49 \\
(3.5)\end{array}$ & $\begin{array}{l}5 \\
(1.1)\end{array}$ & $15(2.2)$ & $\begin{array}{l}3 \\
(1.8)\end{array}$ & \\
\hline $\begin{array}{l}\text { prediagnosed } \\
\text { depression }\end{array}$ & & & $\begin{array}{l}162 \\
(11.6)\end{array}$ & $\begin{array}{l}35 \\
(7.5)\end{array}$ & $\begin{array}{l}101 \\
(14.6)\end{array}$ & $\begin{array}{l}21 \\
(12.4)\end{array}$ & \multirow[t]{3}{*}{$0.001^{b}$} \\
\hline pharmacotherapy ${ }^{\mathrm{e}}$ & & \multirow[t]{2}{*}{28} & $\begin{array}{l}92 \\
(56.0)\end{array}$ & $\begin{array}{l}20 \\
(57.0)\end{array}$ & \multirow{2}{*}{$\begin{array}{l}58(57.0) \\
31(31.0)\end{array}$} & $\begin{array}{l}14 \\
(67.0)\end{array}$ & \\
\hline psychotherapy ${ }^{\mathrm{e}}$ & & & $\begin{array}{l}47 \\
(29.0)\end{array}$ & $\begin{array}{l}13 \\
(37.0)\end{array}$ & & $\begin{array}{l}3 \\
(14.0)\end{array}$ & \\
\hline \multirow{5}{*}{$\begin{array}{l}\text { Anxiety } \\
\text { (according to } \\
\text { HADS) }\end{array}$} & & \multirow[t]{5}{*}{22} & $\begin{array}{l}903 \\
(65.6)\end{array}$ & $\begin{array}{l}369 \\
(80.6)\end{array}$ & $\begin{array}{l}379 \\
(56.0)\end{array}$ & $\begin{array}{l}101 \\
(61.6)\end{array}$ & \multirow[t]{5}{*}{$<0.001^{a}$} \\
\hline & mild & & $\begin{array}{l}474 \\
(35.5)\end{array}$ & $\begin{array}{l}89 \\
(19.4)\end{array}$ & $\begin{array}{l}299 \\
(44.0)\end{array}$ & $\begin{array}{l}63 \\
(38.4)\end{array}$ & \\
\hline & \multirow{3}{*}{$\begin{array}{l}\text { moderate } \\
\text { severe }\end{array}$} & & $\begin{array}{l}259 \\
(18.8)\end{array}$ & $\begin{array}{l}58 \\
(12.7)\end{array}$ & $\begin{array}{l}158 \\
(23.3)\end{array}$ & $\begin{array}{l}28 \\
(17.1)\end{array}$ & \\
\hline & & & $\begin{array}{l}162 \\
(11.8)\end{array}$ & $\begin{array}{l}29 \\
(6.3)\end{array}$ & $\begin{array}{l}118 \\
(17.4)\end{array}$ & $\begin{array}{l}27 \\
(16.5)\end{array}$ & \\
\hline & & & $\begin{array}{l}53 \\
(3.8)\end{array}$ & $\begin{array}{l}2 \\
(0.4)\end{array}$ & $23(3.2)$ & $\begin{array}{l}8 \\
(4.9)\end{array}$ & \\
\hline
\end{tabular}

a Kruskal-Wallis-Test, ${ }^{\text {b}}$ Chi-square test. $P$-values refer to overall group comparisons of patients with low, moderate and high MG severity. P-values $<0.05$ are marked in bold numbers.

$P T S D=$ posttraumatic stress disorder. ${ }^{e}=\%$ of prediagnosed cases. 


\begin{tabular}{|c|c|c|c|c|c|c|}
\hline \multirow{2}{*}{$\begin{array}{l}\text { self-reported MG } \\
\text { severity, n (\%) }\end{array}$} & \multirow[t]{2}{*}{ missing } & Total & Low & Moderate & High & \multirow[t]{2}{*}{$p$-value } \\
\hline & & 1399 & $\begin{array}{l}469 \\
(33.5)\end{array}$ & $\begin{array}{l}690 \\
(51.9)\end{array}$ & $\begin{array}{l}170 \\
(12.8)\end{array}$ & \\
\hline \multirow{2}{*}{$\begin{array}{l}\text { prediagnosed } \\
\text { anxiety }\end{array}$} & \multirow{2}{*}{$\begin{array}{l}24 \\
397\end{array}$} & \multirow[t]{2}{*}{$\begin{array}{l}80 \\
(5.7)\end{array}$} & \multirow{2}{*}{$\begin{array}{l}14 \\
(3.0)\end{array}$} & \multirow{2}{*}{$\begin{array}{l}52(7.5) \\
29(56.0)\end{array}$} & \multirow{2}{*}{$\begin{array}{l}12 \\
(7.1)\end{array}$} & \multirow[t]{3}{*}{$0.004^{b}$} \\
\hline & & & & & & \\
\hline pharmacotherapy & 415 & $\begin{array}{l}45 \\
(56.0)\end{array}$ & $\begin{array}{l}9 \\
(64.0)\end{array}$ & $17(33.0)$ & $\begin{array}{l}17 \\
(24.0)\end{array}$ & \\
\hline psychotherapy $^{\mathrm{e}}$ & & $\begin{array}{l}21 \\
(26.0)\end{array}$ & $\begin{array}{l}3 \\
(21.0)\end{array}$ & & $\begin{array}{l}1 \\
(1.0)\end{array}$ & \\
\hline \multirow{2}{*}{$\begin{array}{l}\text { PTSD after } \\
\text { myasthenic } \\
\text { crisis/exacerbation }\end{array}$} & \multirow[t]{2}{*}{61} & $\begin{array}{l}68 \\
(12.2)\end{array}$ & \multirow[t]{2}{*}{$\begin{array}{l}4 \\
(3.8)\end{array}$} & \multirow[t]{2}{*}{$39(14.1)$} & \multirow[t]{2}{*}{$\begin{array}{l}11 \\
(11.0)\end{array}$} & \multirow[t]{2}{*}{$0.001^{a}$} \\
\hline & & $\begin{array}{l}4.9 \% \\
\text { of the } \\
\text { total) }\end{array}$ & & & & \\
\hline $\begin{array}{l}\text { Prediagnosed } \\
\text { PTSD }\end{array}$ & 14 & $\begin{array}{l}38 \\
(6.8)\end{array}$ & $\begin{array}{l}4 \\
(3.4)\end{array}$ & $23(7.5)$ & $\begin{array}{l}11 \\
(9.8)\end{array}$ & $0.009^{b}$ \\
\hline \multicolumn{7}{|c|}{$\begin{array}{l}{ }^{a} \text { Kruskal-Wallis-Test, }{ }^{b} \text { Chi-square test. } P \text {-values refer to overall group comparisons of patients with } \\
\text { low, moderate and high } M G \text { severity. } P \text {-values }<0.05 \text { are marked in bold numbers. }\end{array}$} \\
\hline
\end{tabular}

A third of patients with a psychiatric diagnosis were under psychotherapeutic treatment (35.5\%), and more than a half under pharmacotherapy ( $57.8 \%$ ). Antidepressants were taken by $69.2 \%$ of patients with a severe and $64.8 \%$ of patients with a moderate depression according to HADS. In contrast, only $50.0 \%$ with severe and $36.2 \%$ with moderate depression were in psychotherapy. Importantly, the majority of patients who underwent psychotherapy (93.9\%) reported benefiting from it. Sleep difficulties were reported by $63.0 \%$. A restricted sexual life due to their MG were reported by $54.0 \%$ of patients.

The median MG-QoL15 score was 12 (IQR 4-26; Table 1). Linear regression analysis revealed that the selfreported MG severity was strongly associated with higher MG-QoL15 scores. The estimated marginal means for MG-QoL15 score for severe vs. mild self-reported MG severity was 18.26 95\% Cl [15.92; 20.60]; Table 3), suggesting a lower HRQoL in patients with a high MG severity as compared to those with mild $M G$ severity $\left(\operatorname{adj} R^{2}=0.264\right)$. 
Table 3

Associations between self-reported MG severity and MGQOL15 scale scores (z-transformed continuous outcome)

\begin{tabular}{|c|c|c|c|c|c|}
\hline & & $\begin{array}{l}\text { M1: } \\
\text { self-reported } \\
\text { severity } \\
\mathrm{N}=1053\end{array}$ & $\begin{array}{l}\text { M2: } \\
\text { additionally } \\
\text { adjusted } \\
\text { for } \\
\text { depression } \\
\mathrm{N}=1049\end{array}$ & $\begin{array}{l}\text { M3: } \\
\text { additionally } \\
\text { adjusted } \\
\text { for gender } \\
\mathrm{N}=1049\end{array}$ & $\begin{array}{l}\text { M4: additionally } \\
\text { adjusted for other } \\
\text { variables }^{\text {a }} \\
\mathrm{N}=975\end{array}$ \\
\hline \multirow{3}{*}{$\begin{array}{l}\text { Self- } \\
\text { reported } \\
\text { MG severity }\end{array}$} & $\begin{array}{l}\text { moderate } \\
\text { vs. mild }\end{array}$ & $\begin{array}{l}13.16(11.63 \\
-14.69)\end{array}$ & $\begin{array}{l}9.40(8.07- \\
10.72)\end{array}$ & $\begin{array}{l}8.99(7.68- \\
10.30)\end{array}$ & $8.09(6.75-9.44)$ \\
\hline & & & & & \\
\hline & $\begin{array}{l}\text { severe vs. } \\
\text { mild }\end{array}$ & $\begin{array}{l}18.26(15.92 \\
-20.60)\end{array}$ & $\begin{array}{l}15.37 \\
(13.39- \\
17.35)\end{array}$ & $\begin{array}{l}15.09 \\
(13.15- \\
17.04)\end{array}$ & $14.36(12.23-16.48)$ \\
\hline \multirow[t]{3}{*}{$\begin{array}{l}\text { Depression } \\
\text { (HADS) }\end{array}$} & $\begin{array}{l}\text { mild vs. } \\
\text { healthy* }\end{array}$ & - & $\begin{array}{l}11.56(9.85 \\
-13.27)\end{array}$ & $\begin{array}{l}11.67(9.98 \\
-13.35)\end{array}$ & $11.51(9.78-13.23)$ \\
\hline & $\begin{array}{l}\text { moderate } \\
\text { vs. } \\
\text { healthy }\end{array}$ & - & $\begin{array}{l}16.81 \\
(14.79- \\
18.83)\end{array}$ & $\begin{array}{l}16.82 \\
(14.83- \\
18.81)\end{array}$ & $17.00(15.01-19.00)$ \\
\hline & $\begin{array}{l}\text { severe vs. } \\
\text { healthy }\end{array}$ & - & $\begin{array}{l}21.37 \\
(17.71- \\
25.02)\end{array}$ & $\begin{array}{l}21.69 \\
(18.10- \\
25.29)\end{array}$ & $21.05(17.33-24.77)$ \\
\hline gender & $\begin{array}{l}\text { female vs. } \\
\text { male }\end{array}$ & - & - & $\begin{array}{l}3.54(2.36- \\
4.72)\end{array}$ & $2.86(1.48-4.24)$ \\
\hline $\begin{array}{l}\text { other } \\
\text { autoimmune } \\
\text { disorder }\end{array}$ & & & & & $1.58(0.11-3.05)$ \\
\hline $\begin{array}{l}\text { Rituximab or } \\
\text { IVIG intake }\end{array}$ & & & & & $3.83(1.83-5.84)$ \\
\hline thymectomy & & & & & $-0.85(-2.23-0.51)$ \\
\hline Adj $R^{2}$ & & 0,264 & 0,490 & 0,506 & 0,560 \\
\hline
\end{tabular}

Stepwise Linear regression analysis. Estimated marginal means with 95\% Cl for MGQOL 15 scale scores. *healthy=no depression according to HADS

${ }^{a}$ M4: Additionally adjusted for thymectomy, other autoimmune disease, cardiovascular disease, IVIG/Rituximab, myasthenic crisis/exacerbation, duration, disease latency age, education, marital status.

The estimated strength of association for self-reported severity on MG-QoL15 decreased after adjusting for depression, whereas model fit increased. The estimated marginal means of MG-QoL15 score for severe vs. mild self-reported MG severity $=15.3795 \% \mathrm{Cl}[13.39 ; 17.35]$; Adj $\mathrm{R}^{2}=0.490 ;$ Table 3). Depression 
as a comorbidity might partly explain the association between self-reported MG disease severity and HRQoL. Accordingly, in model 2, higher depression categories were significantly associated with higher MG-QoL15 scores, thus with a decreased HRQoL (Fig. 1).

In contrast, adjustment for gender in model 3 and age, education, marital status, myasthenic crisis, duration of disease, disease latency and treatment escalation (IVIG/rituximab) in model 4 only slightly affected the effect estimates of self-reported severity (estimated marginal means of MG-QoL15 score for severe vs. mild self-reported MG severity = 14.36 95\% CI [12.23 16.48]; Adj $R^{2}=0.560$; Table 3). In model 4, female gender was significantly associated with a lower HRQoL (higher MG-QoL15 score values; estimated marginal means of MG-QoL15 scale score for female vs. male patients $=2.8695 \% \mathrm{Cl}[1.48$; 4.24]). The same was observed for escalation therapy with IVIG or Rituximab (estimated marginal means of MG-QoL15 scale score for escalation therapy vs. no escalation therapy $=3.8395 \% \mathrm{Cl}[1.83 ; 5.84])$. In contrast, thymectomy was associated with a higher HRQoL (lower MG-QoL15 score values; estimated marginal means for MG-QoL15 scale score for thymectomy vs. no thymectomy $=-0.8595 \% \mathrm{Cl}[-2.23$; $-0.51])$.

The self-reported disease severity of MG patients was associated with the caregiver burden as measured by the BSFC (estimated marginal means of logarithmized BSFC scale score for severe vs. mild selfreported MG severity= $0.1695 \% \mathrm{Cl}[0.13 ;-0.19)]$; Adj $\mathrm{R}^{2}=0.10$; Table 4). Again, the effect estimates for self-reported disease severity decreased after adjustment for depression according to HADS (estimated marginal means of logarithmized BSFC scale score for severe vs. mild depression $=0.1295 \% \mathrm{Cl}[0.09$; $0.15]$; Adj $R^{2}=0.22$ ), suggesting that depression as a comorbidity of the patient explained in part the association between self-reported disease severity and caregiver burden (adj $R^{2}=0.223$ ). In model 2 , higher depression categories were significantly associated with higher BSFC scale scores (Fig. 2). 
Table 4

Associations between self-reported MG severity and BSFC scale scores (log-transformed continuous outcome)

\begin{tabular}{|c|c|c|c|c|c|}
\hline & & $\begin{array}{l}\text { M1: } \\
\text { self- } \\
\text { reported } \\
\text { severity }\end{array}$ & $\begin{array}{l}\text { M2: } \\
\text { additionally } \\
\text { adjusted } \\
\text { for depression }\end{array}$ & $\begin{array}{l}\text { M3: } \\
\text { additionally } \\
\text { adjusted } \\
\text { for gender }\end{array}$ & $\begin{array}{l}\text { M4: additionally } \\
\text { adjusted for } \\
\text { other variables } \\
\text { N=907 }\end{array}$ \\
\hline & & $\mathrm{N}=997$ & $\mathrm{~N}=991$ & $\mathrm{~N}=989$ & \\
\hline $\begin{array}{l}\text { Self-reported } \\
\text { MG severity }\end{array}$ & $\begin{array}{l}\text { moderate } \\
\text { vs. mild }\end{array}$ & $\begin{array}{l}0.09(0.07 \\
-0.11)\end{array}$ & $\begin{array}{l}0.06(0.04- \\
0.08)\end{array}$ & $\begin{array}{l}0.06(0.04- \\
0.08)\end{array}$ & $\begin{array}{l}0.05(0.03- \\
0.07)\end{array}$ \\
\hline & $\begin{array}{l}\text { severe vs. } \\
\text { mild }\end{array}$ & $\begin{array}{l}0.16(0.13 \\
-0.19)\end{array}$ & $\begin{array}{l}0.12(0.09- \\
0.15)\end{array}$ & $\begin{array}{l}0.12(0.09- \\
0.15)\end{array}$ & $\begin{array}{l}0.11(0.08- \\
0.15)\end{array}$ \\
\hline $\begin{array}{l}\text { Depression } \\
\text { (HADS) }\end{array}$ & $\begin{array}{l}\text { mild vs. } \\
\text { healthy* }\end{array}$ & - & $\begin{array}{l}0.12(0.09- \\
0.14)\end{array}$ & $\begin{array}{l}0.12(0.09- \\
0.14)\end{array}$ & $\begin{array}{l}0.13(0.10- \\
0.15)\end{array}$ \\
\hline & $\begin{array}{l}\text { moderate } \\
\text { vs. healthy }\end{array}$ & - & $\begin{array}{l}0.12(0.10- \\
0.15)\end{array}$ & $\begin{array}{l}0.13(0.10- \\
0.16)\end{array}$ & $\begin{array}{l}0.12(0.09- \\
0.15)\end{array}$ \\
\hline & $\begin{array}{l}\text { severe vs. } \\
\text { healthy }\end{array}$ & - & $\begin{array}{l}0.22(0.17- \\
0.28)\end{array}$ & $\begin{array}{l}0.22(0.17- \\
0.27)\end{array}$ & $\begin{array}{l}0.22(0.16- \\
0.27)\end{array}$ \\
\hline gender & $\begin{array}{l}\text { female vs. } \\
\text { male }\end{array}$ & - & - & $\begin{array}{l}-0.03(-0.50- \\
-0.01)\end{array}$ & $\begin{array}{l}-0.03(-0.05- \\
-0.01)\end{array}$ \\
\hline Thymectomy & & & & & $\begin{array}{l}-0,02(-0,04- \\
0.00))\end{array}$ \\
\hline age & & & & & $\begin{array}{l}-0,00(-0,01- \\
0,00)\end{array}$ \\
\hline Adj $R^{2}$ & & 0,098 & 0,224 & 0,232 & 0,265 \\
\hline $\begin{array}{l}\text { Stepwise line } \\
\text { scale scores. } \\
\text { autoimmune } \\
\text { duration, dise }\end{array}$ & $\begin{array}{l}\text { gression an } \\
\text { althy = no de } \\
\text { lase, cardiov } \\
\text { latency, age }\end{array}$ & $\begin{array}{l}\text { sis. Estimat } \\
\text { ssion accol } \\
\text { ular diseas } \\
\text { ucation, } m\end{array}$ & $\begin{array}{l}\text { narginal means } \\
\text { g to HADS. }{ }^{a} M 4 \\
\text { IIG/Rituximab, } \\
\text { I status. }\end{array}$ & $\begin{array}{l}\text { ith } 95 \% \mathrm{Cl} \text { for I } \\
\text { dditionally adj } \\
\text { asthenic crisis }\end{array}$ & $\begin{array}{l}\text { ansformed BSFC } \\
\text { d for thymectomy, } \\
\text { acerbation, }\end{array}$ \\
\hline
\end{tabular}

Adjusting for gender in model 3 and for the other potentially confounding variables in model 4 showed no substantial change in effect estimates, suggesting that these factors did not have an impact on the association between self-reported MG severity and caregiver burden (Table 4), with the exception of thymectomy having a minor impact.

\section{Discussion}

Our study demonstrates that the prevalence of depression, anxiety disorder and PTSD in MG patients is not only high among MG patients, but also underdiagnosed. Of all MG patients, 81.4\% reported no diagnosed mental health condition. Patients with higher self-reported MG severity reported depression and anxiety disorder more frequently than patients with lower MG severity. Self-reported MG disease 
severity is strongly associated with patients $\mathrm{HRQ}$ oL as well as caregiver burden. Beyond MG severity, depression has negative effects on HRQoL in MG patients as well as on caregivers' burden. In addition to the effect of depression, patients HRQoL as well as caregiver burden were negatively associated by female gender of the patient and positively associated by thymectomy.

The prevalence of depression differed between 14 and $58 \% \cdot(9,20)$, whereas the prevalence of anxiety disorders ranged between 20 and 55\% (21-23)'. This variability can be explained by the considerable heterogeneity of the cohorts, sample sizes, clinical measures and assessment methods, including the diagnostic criteria used. PTSD has been reported in $51 \%$ of MG patients after an episode with respiratory insufficiency. A positive finding in PTSD screening was more likely in patients with higher HADS score values (24). The prevalence of psychiatric comorbidities in the general population in Germany (25) is much lower when compared with our findings in the MG cohort data $(15.4 \% \mathrm{vs}$. $35.5 \%$ for anxiety disorder; $8.2 \%$ vs. $30.8 \%$ for depression, and $2.3 \%$ vs. $4.9 \%$ for PTSD). Interestingly, the PTSD screening revealed similar rates of PTSD in MG patients with a history of an exacerbation and a myasthenic crisis. Compared to the prevalence of anxiety disorder of 24 to $29 \%$ in post-stroke and $22 \%$ in multiple sclerosis patients, MG patients were substantially more often affected (26-28).

Our data based on the HADS and PTSD-7 questionnaire suggests a significant underdiagnosis of depression, anxiety disorder and PTSD in MG patients. MG patients with characteristics of depression, anxiety disorder or PTSD had a corresponding diagnosis in only $23.3 \%, 12.7 \%$ and $19.6 \%$, respectively.

In the largest study to date, conducted before $2010,38.6 \%$ of 1518 patients had diagnosed depression (10), which is three times higher than in our study but close to the prevalence established by HADS. While the other characteristics of the MG patients were very similar to our study and both were conducted in Germany, the difference is likely due to a changed situation in the care of MG patients (previously combined specialty for neurology and psychiatry with now often separated residency tracks). Our study highlights the importance of increasing the awareness of mental health conditions and the necessity of implementing diagnostic measures, such as easy to use screening tools. These tools might help to diagnose and treat comorbid mental health conditions in MG patients. For example, studies have shown, that stroke patients benefit from screening for depression (29).

The simultaneous presence of MG and depression, for example, can be challenging for diagnosing depression in MG patients and the other way around. Thus, depressive symptoms can be misinterpreted as myasthenic symptoms such as fatigue. In contrast, the difficulty in clearly distinguishing symptoms of depression from fatigability and fatigue symptoms of $M G$ might lead to delayed or misdiagnosis in $M G$ patients (30). It has been reported that only one patient out of ten with myasthenic symptoms is getting an adequate MG diagnosis (31). Young women are more likely to get a psychiatric diagnosis, whereas men are more often misdiagnosed with other somatic diseases (32). This is consistent with the finding of our study that women have significantly higher latencies in diagnosis than men (3.0 vs. 1.3 years). This raises the question not only of why women are diagnosed later, but also whether a higher latency of diagnosis increases the risk for depression in women. Obviously, the overlap of myasthenic and

Page $14 / 22$ 
depressive symptoms in MG patients (with or without comorbid depression) leads to difficulties for physicians in making an adequate diagnosis. We demonstrated a strong association between selfreported disease severity and HRQoL, which is largely affected by comorbid depression. This is consistent with the results of a previous study of eighty patients (33). Our results suggest that depression is - amongst the variables we investigated - the most influential factor affecting the perceived MG severity and HRQoL. We cannot rule out that the effect is at least partially inverse, with higher MG severity and lower HRQoL leading to a higher likelihood to develop depression. Female gender is strongly associated with a lower HRQoL corroborating findings of previous studies $(5,34)$. It has also been reported that female gender as well as depression are directly associated with a worse disease severity $(35,36)$. Several factors might explain the gender differences in HRQoL including differences in experiencing and reporting the severity of symptoms in general. Female patients tend to report significantly more physical symptoms and higher symptom severity levels than men (37), which might reduce the HRQoL as shown by our results. Secondly, according to our data, female patients experience a longer latency between onset of symptoms and diagnosis. During this period, circumstances like physician hopping, inadequate therapies, experience of discrimination and humiliation and misdiagnosis due to broad differential diagnosis might be stressful and, thus, affect the HRQoL. The knowledge on the interaction of depression, gender, perceived disease severity, and HRQoL in MG patients is scarce. A recent study with 179 patients showed a moderate correlation between disease severity and depression as well as female gender (35). For other chronic diseases like chronic renal disease, rheumatoid arthritis, and cardiovascular disease depression is a known factor affecting perceived disease severity (38-40).

We observed an improved HRQoL after thymectomy. Consistent with our results, gender differences in HRQoL were abolished in MG patients after thymectomy (34). The burden of disease was lower after thymectomy, and patients had fewer exacerbations and also required less immunosuppressive medication $(41,42)$.

Self-reported disease severity of MG and the caregiver burden are strongly associated. Depression increases the effect of this association, but it also has a direct and negative effect on the caregiver burden. In the normal population, the mental status of a care recipient is associated with the caregiver burden and, more importantly, the caregiver burden was nearly significant in predicting depressive symptoms in caregivers (43). Thus, another important aspect of MG care is the provision of supportive measures not only for patients but also for their caregivers. For example, when caregivers are under greater strain, outpatient care services and psychological assist, especially with systemic therapy, psychoeducation, and self-help groups, can provide relief in the social tension field of families. Female gender of the MG patients is associated with a lower caregiver burden, whereas female caregivers of male MG patients experience a higher burden. In general, female caregivers experience more significant caregiver burden than men (44). The higher strain has been explained by women experiencing more secondary stressors, such as financial and relational problems. Depending on the cultural background and the social norms of gender, women feel more obligated to provide care than men and feel more strain (45). 
Our study has several limitations. Although our analyses are based on a large group of patients, we cannot be confident that our cohort is representative. Most participants patients were members of the national self-help group representing approximately a quarter of all German MG patients. Moreover, we investigated only German MG patients. Although the Global Burden of Disease data from the World Health Organization (WHO) shows comparable prevalence amongst high-income countries like European Countries or the United States (46), our data might not reflect the situation of MG patients in low highincome countries. Importantly, because of the cross-sectional design of our study, we cannot draw conclusions about causality.

Although the HADS is a reliable tool for assessing depression and anxiety, it is not identical to the ICD-10 diagnostic criteria. Prevalence of depression as assessed by HADS might be higher than diagnosed according to ICD-10 criteria (47). For assessing gender-related differences in QoL and disease burden we used a heteronormative approach, which does not consider non-binary gender as well as same-sex partnership. Finally, we used the MG-QoL15 scale and not yet the new slightly revised version (MGQoL15r), which is preferred because of a slightly better performance (48).

\section{Conclusion}

A higher awareness of mental illnesses such as depression or anxiety is urgently needed in MG care. Measures for depression, anxiety, PTSD, and MGQOL15 and MG-ADL should be standard tools in MG care. Female MG patients are at higher risk and might benefit from a systematic approach to mental health status assessment in particular. This strategy should be applied as early as possible in the course of the disease in order to offer appropriate treatment services, such as psychotherapeutic care in the outpatient setting. Our findings suggest that guideline-based treatment of depression can improve perceived illness severity and HRQoL in MG patients and reduce caregiver burden.

\section{Declarations}

Acknowledgements

We thank the German Myasthenia Society (Deutsche Myasthenie Gesellschaft) for improving the questionnaire and the cooperation in contacting their members. We are very grateful to all participants for supporting this project. We thank Jane Thümmler for support in data management and Inken Padberg for statistical advice.

Funding: This study was support by the German Research Foundation (Exc 257 NeuroCure).

Conflict of interest/competing interests:

Sophie Lehnerer has received speaker's honoraria and honoraria for attendance at advisory boards from Alexion. Andreas Meisel received speaker's honoraria from Alexion, Grifols and Hormosan. He received honoraria from Alexion, UCB, MorphoSys and argnx for consulting services and financial research 
support from Octapharma and Alexion. He is chairman of the medical advisory board of the German Myasthenia Gravis Society.

Availability of data and material: The data that support the findings of this study are available on request from the corresponding author.

Authors' contributions: Ulrike Harms and Derin Marbin developed the study design and questionnaire supervised by Andreas Meisel; statistical analyses were performed by Derin Marbin and Sophie K. Piper; Derin Marbin, Ulrike Harms, Sophie Lehnerer and Andreas Meisel interpreted the data; Derin Marbin and Andreas Meisel wrote the manuscript.

Ethics approval: The study has been approved by the ethics committee of Charité University Medicine Berlin (reference number EA1/028/17), and registered at clinicaltrials.gov (NCT03205306).

Consent to participate: Informed consent was obtained from all individual participants included in this study.

Consent for publication: All participants gave written consent regarding publishing their data.

\section{References}

1. Verschuuren JJGM, Palace J, Gilhus NE. Clinical aspects of myasthenia explained. Autoimmunity. 2010 Aug;43(5-6):344-52.

2. Carr AS, Cardwell CR, McCarron PO, McConville J. A systematic review of population based epidemiological studies in Myasthenia Gravis. BMC Neurol [Internet]. 2010;10(1):46. Available from: https://doi.org/10.1186/1471-2377-10-46

3. Gold R, Hohlfeld R, Toyka K V. Progress in the treatment of myasthenia gravis. Ther Adv Neurol Disord. 2008 Sep;1(2):36-51.

4. Thomas CE, Mayer SA, Gungor Y, Swarup R, Webster EA, Chang I, et al. Myasthenic crisis: clinical features, mortality, complications, and risk factors for prolonged intubation. Neurology. 1997 May;48(5):1253-60.

5. Boldingh MI, Dekker L, Maniaol AH, Brunborg C, Lipka AF, Niks EH, et al. An up-date on health-related quality of life in myasthenia gravis -results from population based cohorts. Health Qual Life Outcomes [Internet]. 2015 Aug 1;13:115. Available from: https://www.ncbi.nlm.nih.gov/pubmed/26232146

6. Basta IZ, Pekmezovic TD, Peric SZ, Kisic-Tepavcevic DB, Rakocevic-Stojanovic VM, Stevic ZD, et al. Assessment of health-related quality of life in patients with myasthenia gravis in Belgrade (Serbia). Neurol Sci Off J Ital Neurol Soc Ital Soc Clin Neurophysiol. 2012 Dec;33(6):1375-81.

7. Petersson M, Feresiadou A, Jons D, llinca A, Lundin F, Johansson R, et al. Patient-Reported Symptom Severity in a Nationwide Myasthenia Gravis Cohort: Cross-sectional Analysis of the Swedish GEMG Study. Neurology. 2021 Aug;97(14):e1382-91. 
8. Qiu L, Feng H, Huang X, Mo R, Ou C, Luo C, et al. [Study of incidence and correlation factors of depression, anxiety and insomnia in patients with myasthenia gravis]. Zhonghua Yi Xue Za Zhi. 2010 Dec;90(45):3176-9.

9. Suzuki Y, Utsugisawa K, Suzuki S, Nagane Y, Masuda M, Kabasawa C, et al. Factors associated with depressive state in patients with myasthenia gravis: a multicentre cross-sectional study. BMJ Open [Internet]. 2011;1(2):e000313. Available from: http://www.pubmedcentral.nih.gov/articlerender.fcgi? artid $=3244661 \&$ tool=pmcentrez\&rendertype $=$ abstract

10. Twork S, Wiesmeth S, Klewer J, Pöhlau D, Kugler J. Quality of life and life circumstances in German myasthenia gravis patients. Health Qual Life Outcomes [Internet]. 2010;8(1):129. Available from: http://dx.doi.org/10.1186/1477-7525-8-129

11. Winter Y, Schepelmann K, Spottke AE, Claus D, Grothe C, Schröder R, et al. Health-related quality of life in ALS, myasthenia gravis and facioscapulohumeral muscular dystrophy. J Neurol [Internet]. 2010;257. Available from: http://dx.doi.org/10.1007/s00415-010-5549-9

12. Law C, Flaherty C V, Bandyopadhyay S. A Review of Psychiatric Comorbidity in Myasthenia Gravis. Cureus. 2020 Jul;12(7):e9184.

13. Kanner AM. Should neurologists be trained to recognize and treat comorbid depression of neurologic disorders? Yes. Epilepsy Behav. 2005 May;6(3):303-11.

14. Hinz A, Schwarz R, Herrmann C, Buss U, Snaith R. Hospital Anxiety and Depression Scale - Deutsche Version(HADS-D). Diagnostica. 2002 Apr 1;48:112-3.

15. Burns TM, Grouse CK, Wolfe GI, Conaway MR, Sanders DB. The MG-QOL15 for following the healthrelated quality of life of patients with myasthenia gravis. Muscle Nerve. 2011 Jan;43(1):14-8.

16. Breslau N, Peterson EL, Kessler RC, Schultz LR. Short screening scale for DSM-IV posttraumatic stress disorder. Am J Psychiatry. 1999 Jun;156(6):908-11.

17. Maercker A, Forstmeier S, Wagner B, Glaesmer H, Brähler E. Posttraumatische Belastungsstörungen in Deutschland. Nervenarzt [Internet]. 2008;79(5):577. Available from: https://doi.org/10.1007/s00115-008-2467-5

18. FA M. Freiburger Fragebogen zur Krankheitsverarbeitung. RVerres, ed Psychosoz Onkol Springer Verlag Berlin Heidelb.

19. Graessel E, Berth H, Lichte T, Grau H. Subjective caregiver burden: validity of the 10-item short version of the Burden Scale for Family Caregivers BSFC-s. BMC Geriatr. 2014 Feb;14:23.

20. Doering S, Henze T, Schussler G. Coping With Myasthenia Gravis and Implications for Psychotherapy. Arch Neurol [Internet]. 1993;50. Available from: http://dx.doi.org/10.1001/archneur.1993.00540060055018

21. Hoffmann S, Ramm J, Grittner U, Kohler S, Siedler J, Meisel A. Fatigue in myasthenia gravis: risk factors and impact on quality of life. Brain Behav. 2016 Oct;6(10):e00538.

22. Ybarra M, Kummer A, Comini-Frota E, Oliveira J, Gomez R, Teixeira A. Psychiatric disorders in myasthenia gravis. Arq Neuropsiquiatr. 2011 Apr 1;69:176-9. 
23. Lundeen J, Fisher J, Kothari MJ. Frequency of anxiety in myasthenia gravis. J Clin Neuromuscul Dis. 2004 Sep;6(1):9-12.

24. Liu C, Li T, Wang Q, Xu A, Wu B. Post-traumatic stress disorder symptoms after respiratory insufficiency in patients with myasthenia gravis. Psychol Health Med [Internet]. 2020 Sep 2;1-7. Available from: https://doi.org/10.1080/13548506.2020.1807577

25. Jacobi F, Höfler M, Strehle J, Mack S, Gerschler A, Scholl L, et al. Psychische Störungen in der Allgemeinbevölkerung. Studie zur gesundheit erwachsener in Deutschland und ihr zusatzmodul psychische gesundheit (DEGS1-MH). Nervenarzt. 2014;85(1):77-87.

26. Rafsten L, Danielsson A, Sunnerhagen KS. Anxiety after stroke: A systematic review and metaanalysis. J Rehabil Med. 2018 Sep;50(9):769-78.

27. Pérez-Piñar M, Ayerbe L, González E, Mathur R, Foguet-Boreu Q, Ayis S. Anxiety disorders and risk of stroke: A systematic review and meta-analysis. Eur Psychiatry. 2017 Mar;41:102-8.

28. Boeschoten RE, Braamse AMJ, Beekman ATF, Cuijpers P, van Oppen P, Dekker J, et al. Prevalence of depression and anxiety in Multiple Sclerosis: A systematic review and meta-analysis. J Neurol Sci. 2017 Jan;372:331-41.

29. Rogers SC. Poststroke Depression Screening: An Executive Summary. J Neurosci Nurs J Am Assoc Neurosci Nurses. 2017 Apr;49(2):66-8.

30. Kulaksizoglu IB. Mood and anxiety disorders in patients with myasthenia gravis: aetiology, diagnosis and treatment. CNS Drugs [Internet]. 2007;21. Available from: http://dx.doi.org/10.2165/00023210200721060-00004

31. Perez-Nellar J, Rodriguez A. [False negatives in the diagnosis of myasthenia gravis]. Rev Neurol. 2000 Apr;30(8):712-5.

32. Rohr W. Myasthenia gravis in the diagnostic frontier area of psychiatry. Psychiat Prax. 1992;19.

33. Braz NFT, Rocha NP, Vieira ÉLM, Barbosa IG, Gomez RS, Kakehasi AM, et al. Muscle strength and psychiatric symptoms influence health-related quality of life in patients with myasthenia gravis. $J$ Clin Neurosci Off J Neurosurg Soc Australas. 2018 Apr;50:41-4.

34. Lee I, Kaminski HJ, Xin H, Cutter G. Gender and quality of life in myasthenia gravis patients from the myasthenia gravis foundation of America registry. Muscle Nerve. 2018 Feb;

35. Bogdan A, Barnett C, Ali A, AlQwaifly M, Abraham A, Mannan S, et al. Chronic stress, depression and personality type in patients with myasthenia gravis. Eur J Neurol. 2020 Jan;27(1):204-9.

36. Fan X, Xing C, Yang L, Wang J, Feng L. Fatigue, self-efficacy and psychiatric symptoms influence the quality of life in patients with myasthenia gravis in Tianjin, China. J Clin Neurosci Off J Neurosurg Soc Australas. 2020 Sep;79:84-9.

37. Kroenke K, Spitzer RL. Gender differences in the reporting of physical and somatoform symptoms. Psychosom Med. 1998;60(2):150-5.

38. Sacks CR, Peterson RA, Kimmel PL. Perception of Illness and Depression in Chronic Renal Disease. Am J Kidney Dis [Internet]. 1990 Jan 1;15(1):31-9. Available from: https://doi.org/10.1016/S0272- 
6386(12)80589-0

39. Murphy H, Dickens C, Creed F, Bernstein R. Depression, illness perception and coping in rheumatoid arthritis. J Psychosom Res. 1999 Feb;46(2):155-64.

40. Steca P, Greco A, Monzani D, Politi A, Gestra R, Ferrari G, et al. How does illness severity influence depression, health satisfaction and life satisfaction in patients with cardiovascular disease? The mediating role of illness perception and self-efficacy beliefs. Psychol Health [Internet]. $2013 \mathrm{Jul}$ 1;28(7):765-83. Available from: https://doi.org/10.1080/08870446.2012.759223

41. Wolfe GI, Kaminski HJ, Aban IB, Minisman G, Kuo H-C, Marx A, et al. Randomized Trial of Thymectomy in Myasthenia Gravis. N Engl J Med [Internet]. 2016 Aug 10;375(6):511-22. Available from: https://doi.org/10.1056/NEJMoa1602489

42. Rückert JC, Swierzy M, Kohler S, Meisel A, Ismail M. Thymektomie bei Myasthenia gravis TT Thymectomy in Myasthenia gravis. Aktuelle Neurol. 2018;45(04):263-70.

43. Sherwood PR, Given CW, Given BA, von Eye A. Caregiver Burden and Depressive Symptoms: Analysis of Common Outcomes in Caregivers of Elderly Patients. J Aging Health [Internet]. 2005 Apr 1;17(2):125-47. Available from: https://doi.org/10.1177/0898264304274179

44. Miech RA, Shanahan MJ. Socioeconomic Status and Depression over the Life Course. J Health Soc Behav [Internet]. 2000;41(2):162-76. Available from: http://www.jstor.org/stable/2676303

45. Bjelland I, Krokstad S, Mykletun A, Dahl A, Tell G, Tambs K. Does higher education protect against anxiety and depression? The HUNT study. Vol. 66, Social science \& medicine (1982). 2008. 1334$1345 \mathrm{p}$.

46. Lancet [Internet]. 2018 Nov 10;392(10159):1789-858. Available from: https://doi.org/10.1016/S0140-6736(18)32279-7

47. Stoppe G, Bramesfeld A, Schwartz F-W. Volkskrankheit Depression? Bestandsaufnahme und Perspektiven. Dtsch Arztebl Int [Internet]. 2006 Jun 2;103(22):A-1557. Available from: https://www.aerzteblatt.de/int/article.asp?id=51635

48. Burns TM, Sadjadi R, Utsugisawa K, Gwathmey KG, Joshi A, Jones S, et al. International clinimetric evaluation of the MG-QOL15, resulting in slight revision and subsequent validation of the MGQOL15r. Muscle Nerve. 2016 Dec;54(6):1015-22.

\section{Figures}




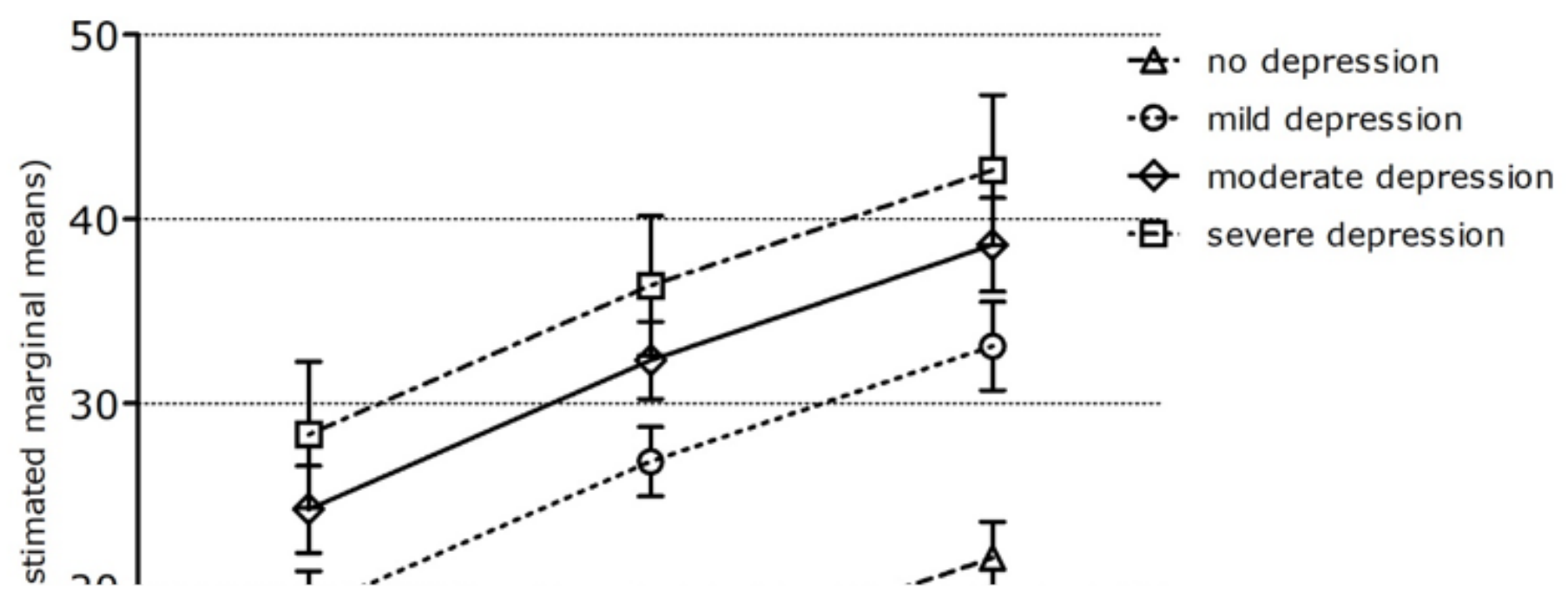

Figure 1

\section{Adjusted MGQOL15 scale scores for different severities of depression according to HADS}

Estimated marginal means with 95\% Cl for MGQOL 15 scale scores after full adjustment for depression, gender, thymectomy, other autoimmune disease, cardiovascular disease, IVIG/Rituximab, myasthenic crisis/exacerbation, duration, disease latency age, education, marital status. MGQOL15=Myasthenia gravis Quality of Life Score, short version; HADS= Hospital Anxiety and Depression Scale 


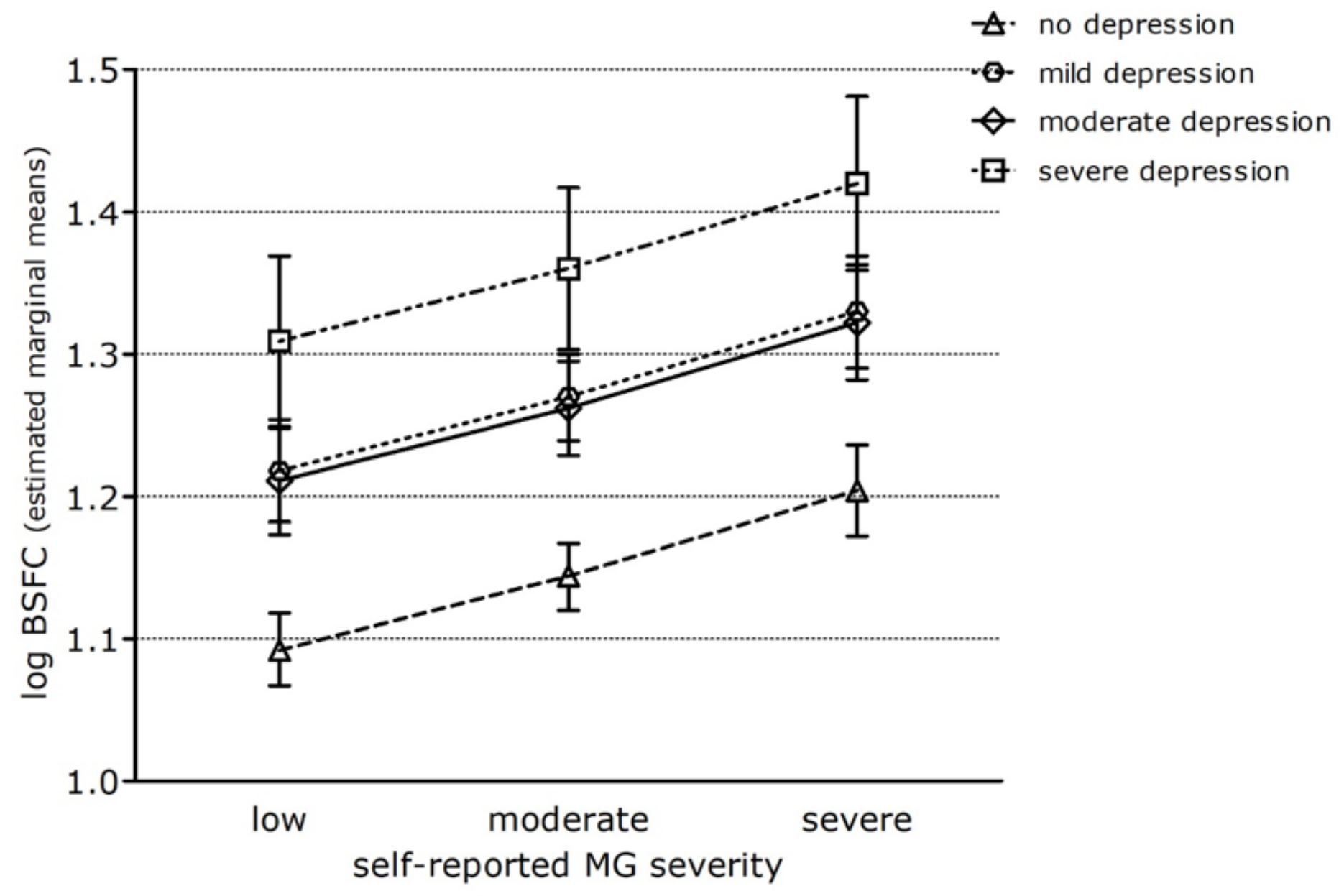

Figure 2

Adjusted logarithmized BSFC scale scores for different categories of depression according to HADS

Estimated marginal means with $95 \% \mathrm{Cl}$ for logarithmized BSFC scale scores after full adjustment for depression, gender, thymectomy, other autoimmune disease, cardiovascular disease, IVIG/Rituximab, myasthenic crisis/exacerbation, duration, disease latency age, education, marital status. BSFC=burden scale for family caregivers; HADS= Hospital Anxiety and Depression Scale 